\title{
Finite Element Based HWB Centerbody Structural Optimization and Weight Prediction
}

\author{
Frank H. Gern ${ }^{1}$ \\ NASA Langley Research Center, Hampton, VA 23681
}

\begin{abstract}
This paper describes a scalable structural model suitable for Hybrid Wing Body (HWB) centerbody analysis and optimization. The geometry of the centerbody and primary wing structure is based on a Vehicle Sketch Pad (VSP) surface model of the aircraft and a FLOPS compatible parameterization of the centerbody. Structural analysis, optimization, and weight calculation are based on a Nastran finite element model of the primary HWB structural components, featuring centerbody, mid section, and outboard wing. Different centerbody designs like single bay or multi-bay options are analyzed and weight calculations are compared to current FLOPS results. For proper structural sizing and weight estimation, internal pressure and maneuver flight loads are applied. Results are presented for aerodynamic loads, deformations, and centerbody weight.
\end{abstract}

\section{Nomenclature}

$\begin{array}{ll}\text { AEDC 16T } & =\text { Arnold Engineering Development Center's 16ft transonic wind tunnel } \\ \text { BWB } & =\text { Blended Wing Body } \\ C_{D} & =\text { Total drag coefficient } \\ C_{L} & =\text { Total lift coefficient } \\ C_{M} & =\text { Total moment coefficient } \\ \text { CFD } & =\text { Computational Fluid Dynamics } \\ \text { DLM } & =\text { Doublet Lattice Method } \\ \text { ERA } & =\text { Environmentally Responsible Aviation Project at NASA } \\ \text { FEM } & =\text { Finite Element Method } \\ \text { FLOPS } & =\text { Flight Optimization System } \\ \text { HWB } & =\text { Hybrid Wing Body } \\ \text { LE } & =\text { Leading edge } \\ \text { MAC } & =\text { Mean Aerodynamic Chord } \\ \text { OML } & =\text { Outer Mold Line } \\ \text { Re } & =\text { Reynolds number (based on MAC unless stated otherwise) } \\ \text { TE } & =\text { Trailing Edge } \\ \text { TOGW } & =\text { Take-Off Gross Weight } \\ \text { VLM } & =\text { Vortex Lattice Method } \\ \text { VSP } & =\text { Vehicle Sketch Pad } \\ \alpha & =\text { Angle of attack (deg) }\end{array}$

\section{Introduction}

HYBRID wing body (HWB) or Blended Wing Body (BWB) aircraft concepts have been considered promising alternatives to conventional tube and wing configurations due to their large potential fuel savings and increased aerodynamic efficiency. ${ }^{1}$ Fuel burn reductions of $25 \%$ and higher have been published for some ultra-high capacity configurations carrying up to 800 passengers. Most notable is the work of Liebeck and his co-workers at The Boeing Company. Their 450 passenger BWB-4501L design recently resulted in the $8.5 \%$ scale X-48B flight demonstrator (Fig. 1). ${ }^{2}$

\footnotetext{
${ }^{1}$ Senior Aerospace Engineer, Aeronautics Systems Analysis Branch, Member AIAA.
} 
A significant difficulty in dealing with HWB design optimization has always been the lack of a data base of known "flying" designs which may serve as calibration and validation points for optimization programs like FLOPS, especially when compared to the vast amount of available tube and wing aircraft data. When transitioning from the conceptual to the preliminary design phase, the aircraft designer needs to be sure that the design chosen for further optimization is actually a viable design, and as a result of the lack of validation cases, the development of improved fidelity analysis tools becomes imperative for the conceptual design loop.

To validate the projected fuel burn and noise reduction potential of HWB designs for NASA's Environmentally Responsible Aviation (ERA)

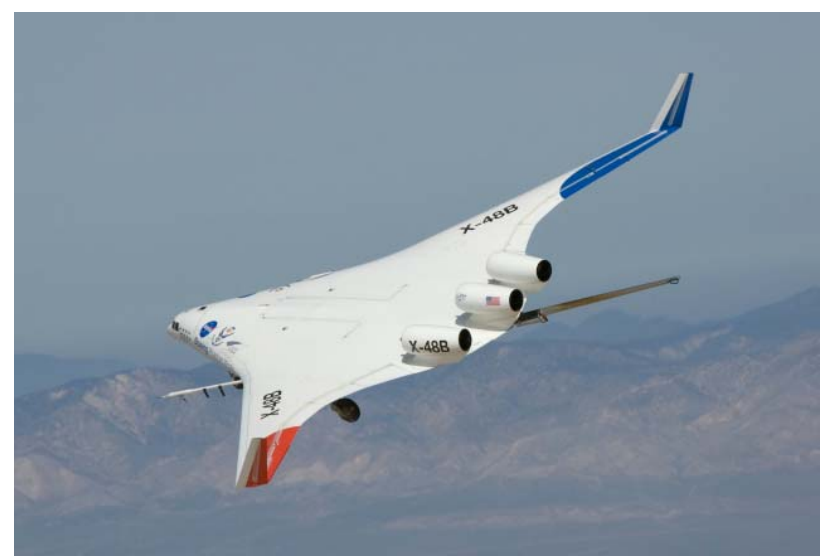

Figure 1: Boeing's X-48B Blended Wing Body flight demonstrator (Boeing picture). project, significant efforts have been put forward to develop advanced structural and aerodynamic analysis tools for HWB conceptual design optimization. Aerodynamic methods improvement has been geared towards increased fidelity in-the-loop methods like enhanced panel codes and computational fluid dynamics (CFD). ${ }^{3}$ Recently developed structures tools include FEM based analyses to provide enhanced capabilities for HWB centerbody sizing and weight estimation.

\section{HWB Centerbody Weight Estimation}

As compared to the fuselage of a conventional tube and wing transport airplane, the complex geometry of an HWB centerbody structure poses unique challenges for structural sizing and weight calculation. Unconventional loading under flight, maneuver, and cabin pressure loads make centerbody sizing and weight estimation in a conceptual design environment difficult, increasing the uncertainty of optimized design predictions in terms of structural weight, fuel burn, and concept efficiency. Furthermore, due to the lack of a database of flight articles for calibration, design sensitivities are unknown and geometric scaling of results from detailed point design analysis becomes questionable.

\section{A. Original FLOPS Based Analyses}

The Flight Optimization System (FLOPS) ${ }^{4}$ software is a multidisciplinary system of computer programs for conceptual and preliminary design and evaluation of advanced aircraft concepts. The modular nature of FLOPS allows the user to incorporate new equations and data tables that enable the program to analyze a wide variety of concepts. The current interest in analyzing transports with an HWB configuration has led to the need for a method to rapidly size and analyze conceptual BWB designs. FLOPS is widely used at NASA for evaluating a variety of configurations.

The original version of FLOPS does not have the ability to accurately represent a HWB configuration, as calculations are based on empirical equations derived from data for conventional transport aircraft. In general, FLOPS assumes cylindrical fuselages and calculates the weight of those tubes based on equations for circular fuselages. The complex geometry and structural loading of an HWB centerbody therefore results in poor weight estimates. FLOPS has been used extensively to analyze the impact of advanced engines and distributed propulsion on HWB configurations, as well as the noise and emissions generated by an HWB. These studies used HWB configurations from more detailed analyses and matched FLOPS results of the weights and performance for the baseline condition by altering input values and overriding internal calculations. While this is sufficient for analyzing the impact of changes to an HWB configuration with accompanying detailed analyses, its usefulness is limited to a particular mission and aircraft size.

\section{B. Enhanced FLOPS Based HWB Centerbody Weight Prediction}

Nickol and McCullers introduced modifications to FLOPS to include an option to layout and size an HWB cabin. ${ }^{5}$ In addition, the FLOPS weight routines were updated with an option to estimate the weight of an HWB. These modifications, combined with propulsion and aerodynamic inputs, enable an HWB mission analysis. The FLOPS user's manual was updated to reflect these changes in the documentation for the weights module. ${ }^{6}$ 
Nickol and McCullers assume that the cabin is shaped like a "home plate" in baseball, with the point at the nose of the aircraft, shown as the shaded area in Fig. 2. This home-plateshaped cabin is sized by assigning an area to each passenger (by using standard packing rules for the number abreast, seat pitch, and passenger class) and allowing for the required number of utility areas (i.e., lavatories, galleys, and closets) based on the number of passengers in each class.

The area that is required for passengers and utilities is combined with the areas for aisles and wasted space which results from the specific geometry, to define the required total cabin area. More detailed packing and area assumptions per passenger class can be found in Refs. 5 and 6.

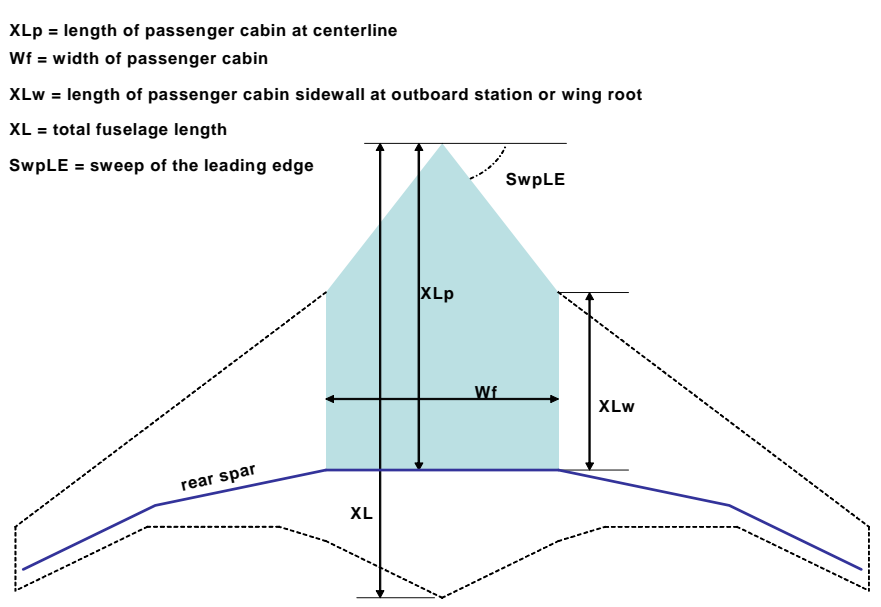

Figure 2: HWB centerbody layout for FLOPS by Nickol and McCullers. ${ }^{5}$

\section{Centerbody Finite Element Analysis by Bradley}
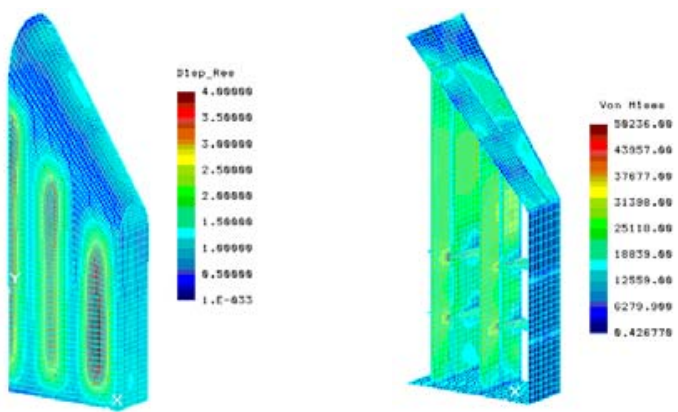

Figure 3: HWB centerbody finite element representation by Bradley. ${ }^{7}$
In an effort to improve HWB centerbody weight predictions, a finite element based approach was implemented by Bradley in 2004. ${ }^{7}$ The purpose of this analysis was not to predict the actual weight of a given centerbody structure, but to find a relationship between TOGW, cabin planform area, and cabin weight. The relationship was determined through manually generated generic centerbody finite element models.

Analysis results were then calibrated to match predictions of a more detailed structural analysis performed by Boeing. The same generic HWB centerbody geometry was used to create a family of five transports, sized for approximately 250 to 450 passengers, at 50-passenger increments.

Data obtained from the finite element analysis was used to find the coefficients of a weight estimate equation in the form

$$
W_{\text {cabin }}=K_{s} * 0.316422(T O G W)^{0.166552}\left(S_{\text {cabin }}\right)^{1.061158}
$$

and a new fuselage design subroutine was added to FLOPS to determine the size of the HWB centerbody. The centerbody dimensions can be entered in an input file to analyze an HWB of known geometry, or the dimensions can be calculated based on the number of passengers. Since the derived regression curve is based on five distinct, though very representative centerbody structural layouts, its sensitivity to changes in centerbody geometry is unknown. Therefore, Bradley's method is best suited for analyses within a given class and mission profile of a specific platform.

\section{Centerbody Finite Element Modeling from VSP Models by Murchison}

To improve the versatility of finite element based centerbody structural analyses, Murchison developed a Matlab based routine in 2010 which extracts centerbody structural features from Vehicle Sketch Pad (VSP) ${ }^{8}$ based HWB models. The program interprets the VSP structural layout, interpolates the unstructured CTRIA elements into more regular CQUAD4 elements, and outputs a Nastran compatible bulk data file (Fig. 4). ${ }^{9}$ The routine still requires the structure to be modeled in VSP.
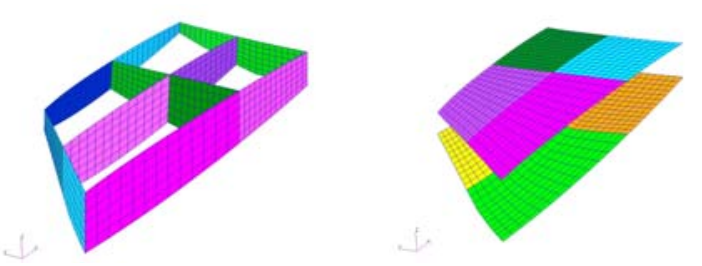

Figure 4: HWB centerbody FEM model based on VSP structural layout. ${ }^{9}$ 


\section{E. Boeing BWB Centerbody Finite Element Analyses}

A detailed vehicle-level BWB finite element model was presented by Li and Velicki from The Boeing Company in Ref. 10. The model features the vehicle's fuselage skins, frames, ribs, spars and floors, wing skins, spars and ribs, vertical stabilizer, movable control surfaces, high-lift devices, and bulkheads. Structural cutouts were included for landing gear doors and cargo doors (Fig. 5). The total number of elements in the vehicle model is approximately 44,000 , representing more than 142,000 degrees of freedom.

Boeing used FAR-25 Airworthiness Standards as guidelines in defining loads criteria and evaluated approximately 80 load
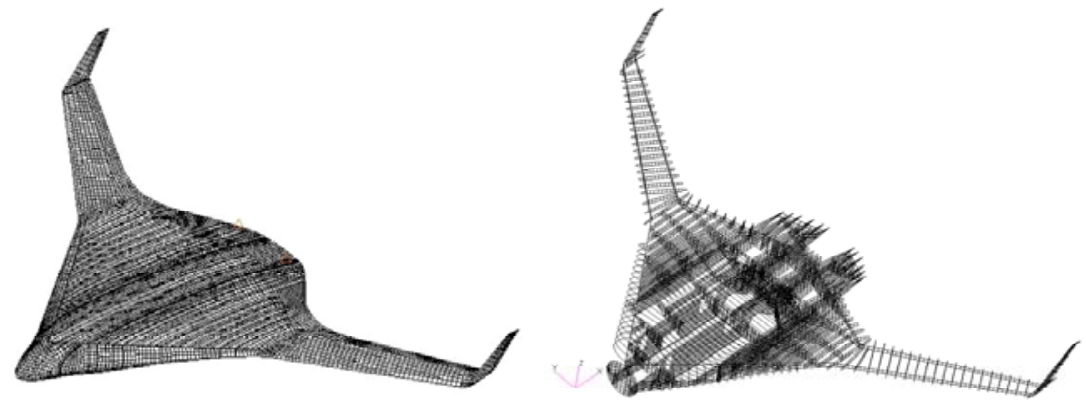

Figure 5: Boeing BWB Finite element model by Li and Velicki. ${ }^{10}$ conditions to determine the critical cases. After the loads analysis, they determined thirteen load cases to be critical for BWB vehicle sizing and optimization. Details of their load cases and analysis are given in Ref. 10. An updated version of this model was also used to implement a PRSEUS pressure cabin and its associated weight savings. Details of the PRSEUS concept can be found in Refs. 11 and 12.

\section{Scalable HWB Centerbody and Wing Box Finite Element Model}

The previously presented methods for structural sizing and weight estimation of an HWB centerbody are either based on regression curve fits covering a family of sizes or missions, or on detailed point design analyses for an already optimized vehicle. However, to investigate the scalability of the HWB structural concept, particularly the centerbody, the present work is intended to close the gap between these different approaches by building a highly scalable finite element based model that captures the geometry, structural layout, and loading of the HWB centerbody in sufficient detail to yield meaningful results for the ongoing HWB scaling study presented by Nickol in Ref. 13.

\section{A. VSP Outer Mold Line Data and Centerbody Parameterization}

Geometry data for the structural analysis is based on VSP outer mold line (OML) data for the HWB vehicle under investigation. The VSP OML is output into a data file as triangularized surface data in terms of CTRIA6 finite elements (Fig. 6).

a)

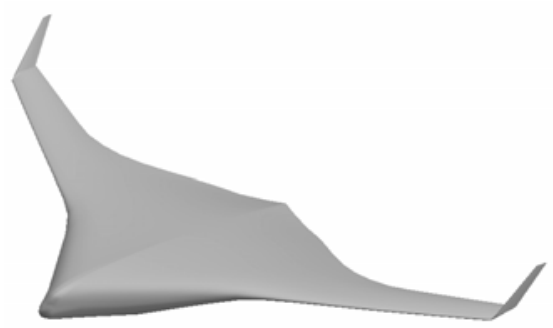

Figure 6: a) VSP model of a generic HWB configuration, b) VSP OML data as a set of triangularized faces of CTRIA6 elements.

To ensure the compatibility of the present structural analysis with previous structural modules implemented in FLOPS, the "home plate" geometry introduced by Nickol and McCullers has been used to define the basic parameters of the centerbody finite element model (see Refs. 5 and 6, Fig. 2). The centerbody "home plate" 
geometry is projected onto the HWB OML data and the centerbody geometry is then created by slicing the OML surface data at the home plate boundaries (Fig. 7).

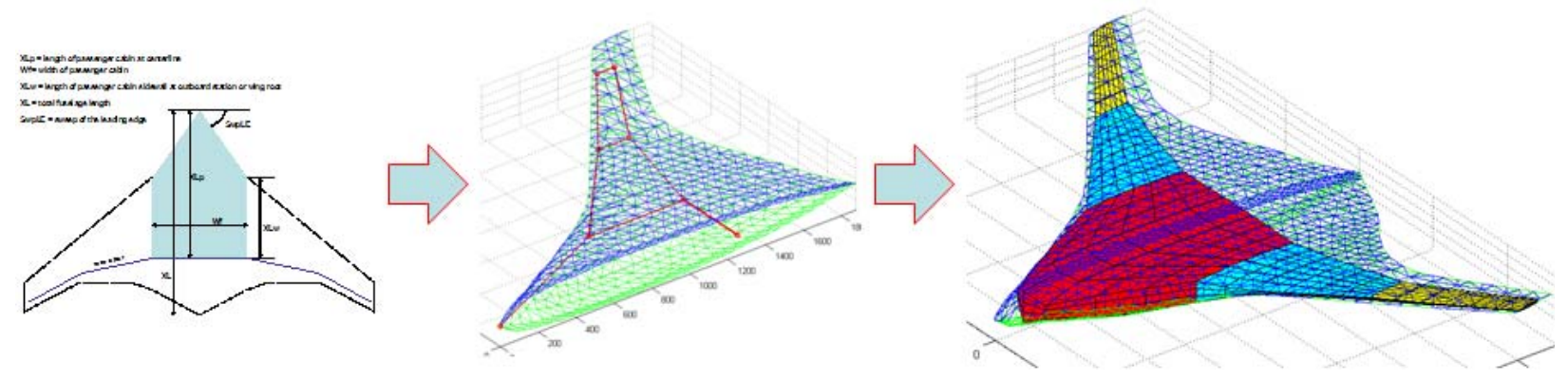

Figure 7: Parametric definition of HWB centerbody and wing structure.

The complete HWB primary structure is represented by three components: centerbody, mid section, and outboard wing (see Fig. 7). The mid section and outboard wing of the HWB are added to the structural model to allow for proper application of wing lift and moment distributions on the centerbody. The chordwise locations of front and rear spars for both mid section and outboard wing are assumed to be at $12.5 \%$ and $62.5 \%$, respectively. The front bulk head of the centerbody is defined by standard cockpit size assumptions. All three primary structural components are then modeled as CQUAD4 finite elements in Nastran. The complete design model features front spars, rear spars, skin sections, side walls, and internal walls if applicable.

\section{B. HWB Centerbody Structure Options}

Three different centerbody design options are available for structural analysis and optimization (Fig. 8). The basic single bay centerbody design does not feature any internal walls and is suitable for fast centerbody weight estimation. However, displacements due to the applied loads are not represented correctly and the introduction of displacement constraints into the design model proved difficult. The three- and five-bay centerbody designs represent actual centerbody structures from preliminary design studies like the one presented in Ref. 10 more closely. The internal walls provide significant displacement relief and allow for an accurate application of displacement constraints in the design model.
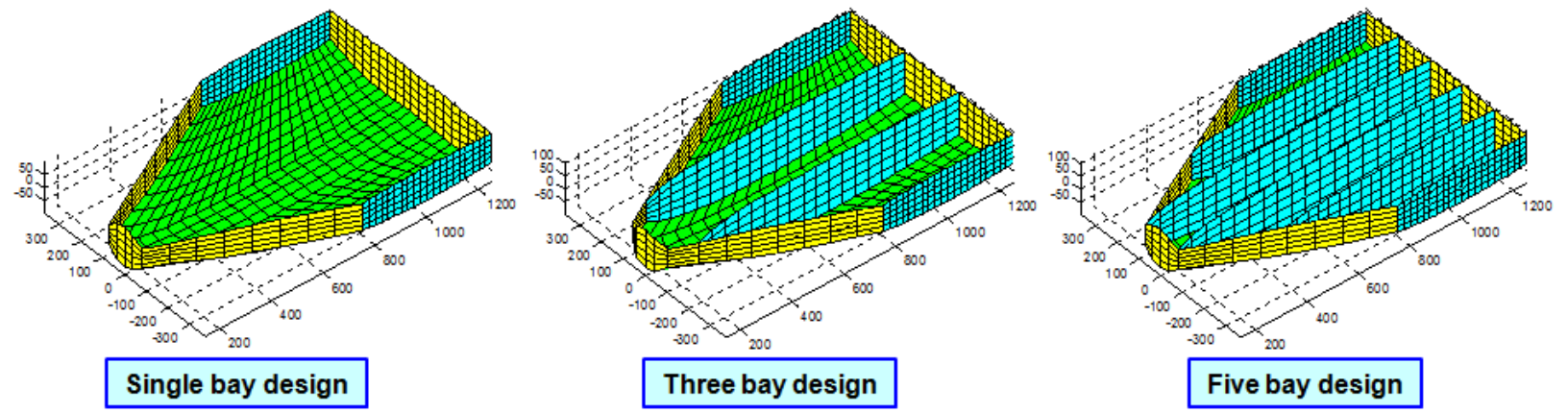

Figure 8: HWB Centerbody design options for structural analysis and optimization.

As depicted in Fig. 8, the non-pressurized section aft of the HWB centerbody is currently not included in the primary structure finite element model, since it is assumed that the majority of the wing bending moment is carried through the centerbody structure. The FLOPS modifications outlined in Ref. 5 already account for the aft section in a separate weight term. However, due to the modular nature of the structural representation, a future incorporation of the HWB aft section into the finite element model will be straight forward and easy to implement.

\section{Stiffened Panel Modeling}

Researchers at The Boeing Company in Huntington Beach, California have been developing highly-integrated stitched-composite airframe structures tailored and optimized to exploit the orthotropic nature and processing advantages of carbon fiber materials. The Pultruded Rod Stitched Efficient Unitized Structure (PRSEUS) concept 


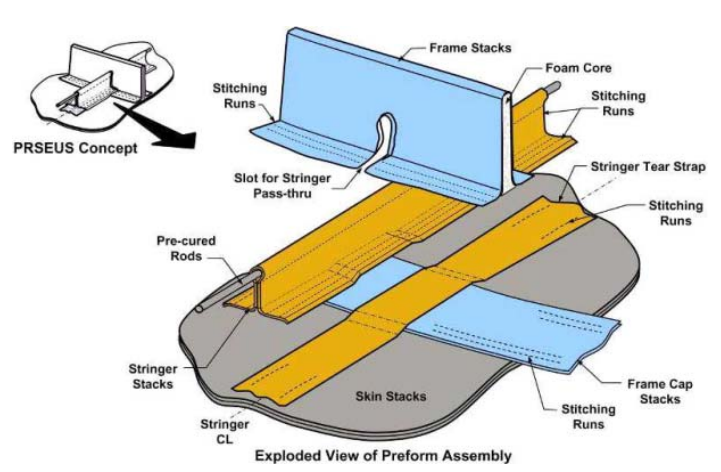

Figure 9: The PRSEUS structural concept. ${ }^{10}$

departs from conventional laminated composite design practices, manufacturing processes, and tooling techniques. This leads to substantial reductions in manufacturing and assembly costs while yielding the higher levels of structural performance that are critical for BWB airframes (Fig. 9). ${ }^{10-12}$ The PRSEUS approach is deemed necessary for the BWB because it enables the flat-sided pressure cabin to be weightcompetitive with traditional circular fuselage shells and their state-of-the-art no-growth design methods.

Modeling stiffened panels, and even more so the PRSEUS structural concept, in a conceptual design environment presents significant challenges in terms of FEM model size and complexity. However, in order to realistically apply displacement constraints for design optimization, the overall stiffness of the structural components must be matched. For this purpose, the 12I/T**3 entry in the Nastran PSHELL card has been modified from its default value of 1 to a value reflecting the actual bending moment of inertia of the PRSEUS panels vs. the bending stiffness of a homogenous shell. ${ }^{14}$

\section{Aeroelastic Modeling}

To apply maneuver loads for structural sizing, the original VSP geometry is sliced at given spanwise locations to obtain planform geometries for the Nastran CAERO Doublet Lattice panels. Wing twist and camber distributions are extracted from the VSP airfoil geometry data and applied to the aerodynamic panels as a fixed downwash. Aerodynamic loads are then splined onto the front and rear spars to accurately apply forces and moments to the
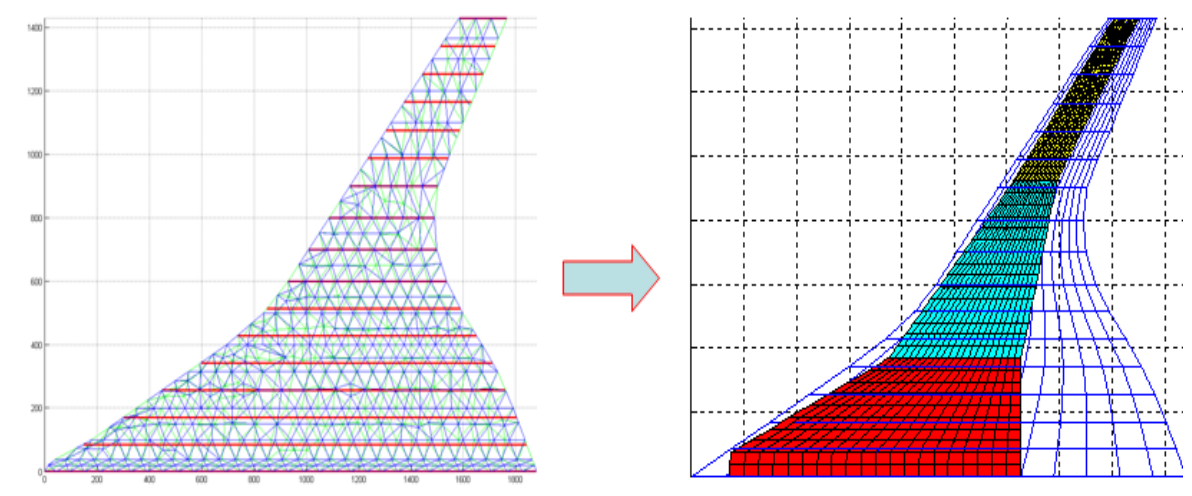

Figure 10: Aerodynamic slicing of VSP OML data and aerodynamic paneling with underlying structural model in Nastran. centerbody (Fig. 10).

A common approach in applying maneuver loads to the structure is to allow Nastran to trim the aircraft to the correct pitch attitude for a given load factor in the TRIM card. ${ }^{15}$ However, in the present analysis, the gross weight

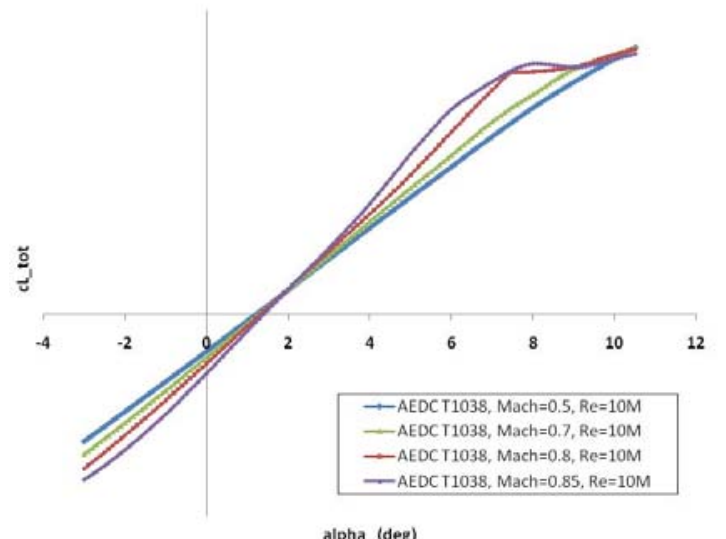

Figure 11: Lift coefficient data from $X-48 B$ wind tunnel testing for maneuver load interpolation. ${ }^{16,17}$ of the aircraft is unknown, rendering it impossible to find the correct pitch trim this way. As an alternative, the pitch angle of the aircraft is determined by extrapolation using a generic lift curve that is applicable to a wide variety of HWB configurations. In June 2007, the X-48B 2\% scale model of the BWB-450-1L was tested at the Arnold Engineering Development Center (AEDC) 16T wind tunnel. ${ }^{16,17}$ The test was called TF1038 under the AEDC designation system. As a result of this test, sufficient data was generated to develop a transonic aerodynamic database for vehicle lift, drag, and moment coefficients vs. angle of attack and Mach number.

Data from this test was used to interpolate to the correct maneuver load conditions based on the known cruise lift coefficient, Mach number and altitude from a FLOPS analysis (Fig. 11). The resulting dynamic pressure is then multiplied by the respective maneuver load factor (e.g. 2.5 for the $2.5 \mathrm{~g}$ maneuver) to apply the correct maneuver loads.

6

American Institute of Aeronautics and Astronautics 
This approach generally slightly overpredicts structural maneuver loads, as inertia relief from the nonstructural masses is not captured, resulting in conservative centerbody weight estimates.

\section{E. Load Cases for Structural Sizing}

Loads assumptions for commercial transport aircraft are defined in FAR-25 (Federal Aviation Regulations Part 25 - Airworthiness Standards: Transport Category Aircraft). To fully satisfy these regulations, the airframe manufacturer generally has to analyze thousands of different load cases throughout the flight envelope to identify the critical ones. Only a subset of critical load cases is used in preliminary design, with an even smaller subset of only a few load cases being used for conceptual design optimization. A comprehensive overview and load case development of design critical load cases for BWB platforms is presented in Ref. 18. The three most significant load cases for sizing the HWB centerbody have been identified in Ref. 18 as

- $\quad$ 2.5-g limit load (centerbody and wingbox)

- $\quad$-1.0-g limit load (centerbody and wingbox)

- 1.33 P internal pressure limit load (centerbody only)

A safety factor of 1.5 has been applied to the limit load stress margins to account for ultimate loads. Only symmetric load conditions are currently implemented, therefore only a half-model is required for analysis and design optimization.

\section{F. Nastran Design Model}

Nastran's Solution 200 (SOL200) is used for structural analysis and design optimization. ${ }^{19}$ In SOL200, the design optimization is multi-disciplinary in the sense that the optimization for all load cases is performed simultaneously. The centerbody weight is used as an objective function to be minimized.

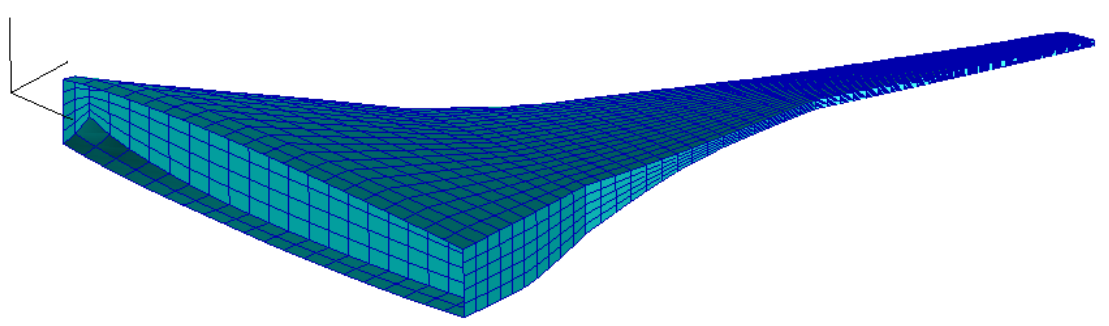

Figure 12: FEM design model of the HWB centerbody and wing structure.

Design variables are the individual panel thicknesses of the centerbody elements. Thickness constraints are placed on the design variables to account for minimum gauge limitations. Stress constraints are based on PRSEUS material properties. Displacement constraints are based on maximum allowable deformations to maintain laminar flow and overall aerodynamic flow quality. The model is fully scalable and allows for arbitrary numbers of elements in the $\mathrm{x}, \mathrm{y}$, and z-directions. However, typical design models for the present analyses have been found to work well with 2,500 to 3,500 CQUAD4 elements, resulting in 15,000 to 20,000 degrees of freedom. Optimizing the centerbody only results in 700 to 900 design variables, with the same number of thickness constraints and 600 to 800 displacement constraints.

\section{G. Centerbody Weight Calculation}

The centerbody structural weight is automatically calculated by Nastran when using weight as an objective function. To obtain the total centerbody weight, the PRSEUS material density of $0.057 \mathrm{lb} / \mathrm{in}^{3}$ is multiplied by a correction factor accounting for weight penalties due to production breaks, the centerbody leading edge, main and cargo deck floors, doors and supports, maintenance platforms, lightning protection, as well as paint, primer, and sealant. A front bulkhead simulates a closed pressure vessel for the 1.33P internal pressure load without the need to model the complex cockpit geometry, while still applying realistic loads to the centerbody front section. The combined weight of cockpit and nose is assumed to be constant at 2000lbs. Generally, this number does not vary significantly with aircraft size due to commonality requirements of modern aircraft family designs.

The objective of the current analysis is to increase the fidelity of structural centerbody weight predictions. As a result, the density of wingbox and midsection are set to zero to only generate the centerbody weight in Nastran. FLOPS numbers have been used for wingbox and midsection weights in the HWB scaling study from Ref. 13. However, by simply defining the correct densities for wingbox and midsection materials, and by adding the respective elements to the design model, a complete structural optimization and weight calculation of the overall HWB primary structure can be performed. 


\section{Model Validation}

\section{A. Centerbody Weight Validation}

A centerbody weights prediction tool developed by The Boeing Company was used to validate the centerbody weight calculations from the finite element model. A BWB450 type centerbody design was chosen to generate a suitable centerbody geometry using the home plate approach described previously. Figs. 13 and 14 show deformation results for the initial and optimized centerbody configuration for both the 1.33P internal pressure load and the 2.5-g maneuver load case. Note that due to the absence of internal walls, deformations for the internal pressure case from the stress-based model are unrealistic.

a) Initial configuration

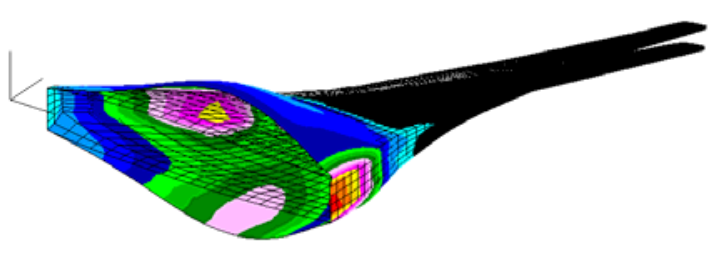

b) Optimized configuration

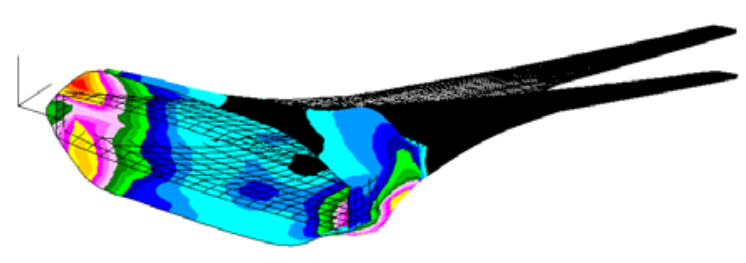

Figure 13: 1.33P internal pressure load deformations (not to scale). a) Initial configuration

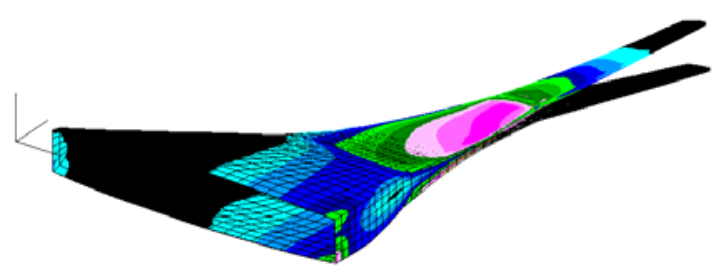

b) Optimized configuration

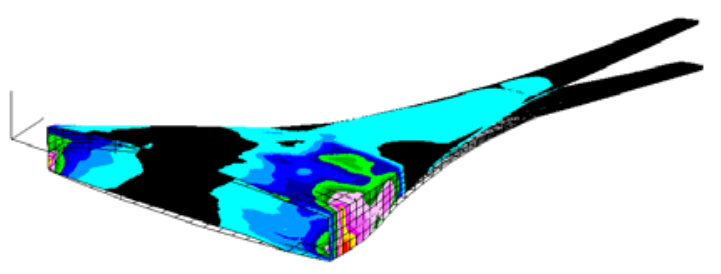

Figure 14: 2.5-g maneuver load deformations (not to scale).

Convergence for the stress-based model is fast (Fig. 15). Therefore, the stress-based model is suitable for short turnaround times, when a quick yet relatively accurate centerbody weight estimate is needed without the application of displacement constraints. Model scalability was checked by using different numbers of CQUAD4 elements on the $\mathrm{x}, \mathrm{y}$, and z-directions, resulting in centerbody FEM models of 256, 480, 750, and 1000 elements. Figure 15 illustrates that weight convergence is almost independent of model size, indicating excellent scalability of the model.

To investigate the influence of the individual load cases on the overall centerbody weight, individual optimizations were performed for each of the three load cases. A comparison of convergence histories for the individual analyses with the results from a simultaneous optimization of all load cases shows that the centerbody is almost entirely sized by the 1.33P internal pressure condition, as the weight obtained from the pressure load case is almost identical to the weight obtained from a simultaneous optimization (Fig. 16).

Centerbody weights obtained by applying only the maneuver load cases result in much lower numbers, with the majority of the element thicknesses running all the way down to minimum gauges. Closer examination of the results shows that only a small region of the rear spar is sized by the 2.5-g maneuver load.

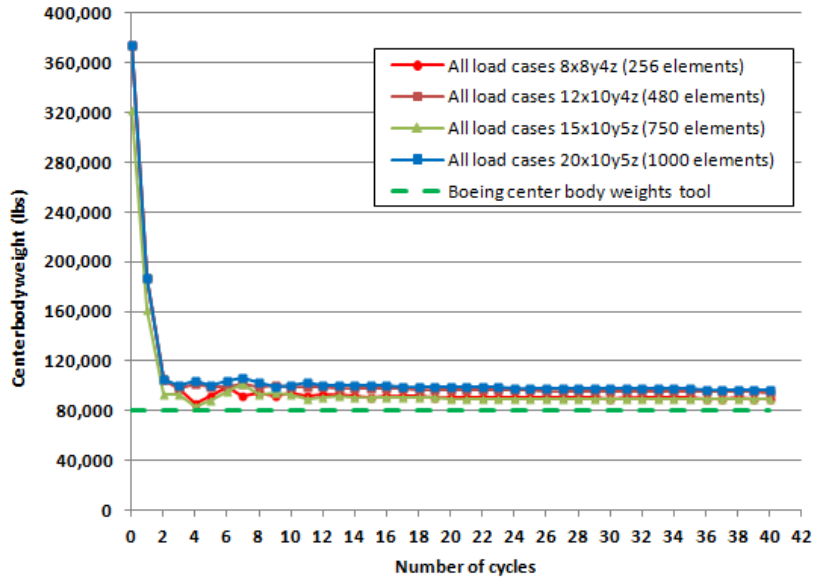

Figure 15: Convergence history and scalability check for different numbers of centerbody finite elements.

American Institute of Aeronautics and Astronautics 


\section{B. Multi-Bay Designs}

As already mentioned, centerbody designs featuring internal walls are more representative of more detailed preliminary design studies. The internal walls provide significant displacement relief for the centerbody walls under the internal pressure load. The more realistic stress and strain distributions in the structure lead to more realistic optimization results.

This is of particular importance for scaling studies, where centerbody designs from 98passenger sizes to 400-passenger sizes were studied. Three and five-bay designs were investigated (see Fig. 8). A four bay design was not considered, as an internal wall in the centerline of the vehicle was deemed to lead to challenging structural issues in the front section of the centerbody where the cockpit connects to the front bulkhead.

a) Three-bay design

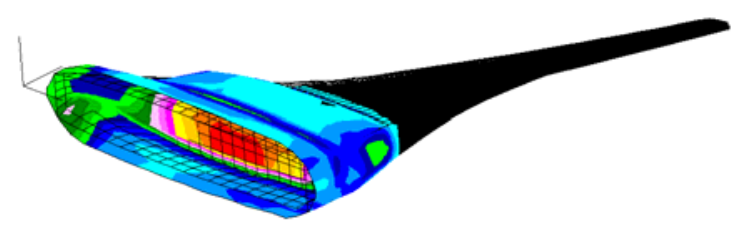

b) Five-bay design

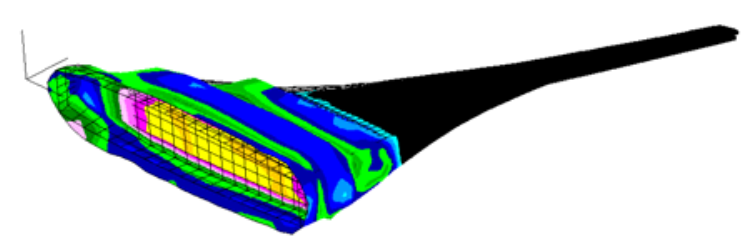

Figure 17: 2P internal pressure load deformations for multi-bay centerbodies (not to scale).

\section{Spanload Verification}

For aerodynamic spanload verification, the Nastran maneuver loads have been compared to Vorview $^{20,21}$ results from a previous study. ${ }^{3}$ Wind tunnel test data in terms of total lift, moment, and drag coefficients was available from the transonic test at AEDC described in section III.D ${ }^{16,17}$.

Spanload distributions from Nastran were validated by comparing spanwise lift coefficients with results from a Vorview vortex lattice analysis on the same geometry. The Vorview results had been previously validated using the total lift, drag, and moment coefficients from the AEDC wind tunnel test.

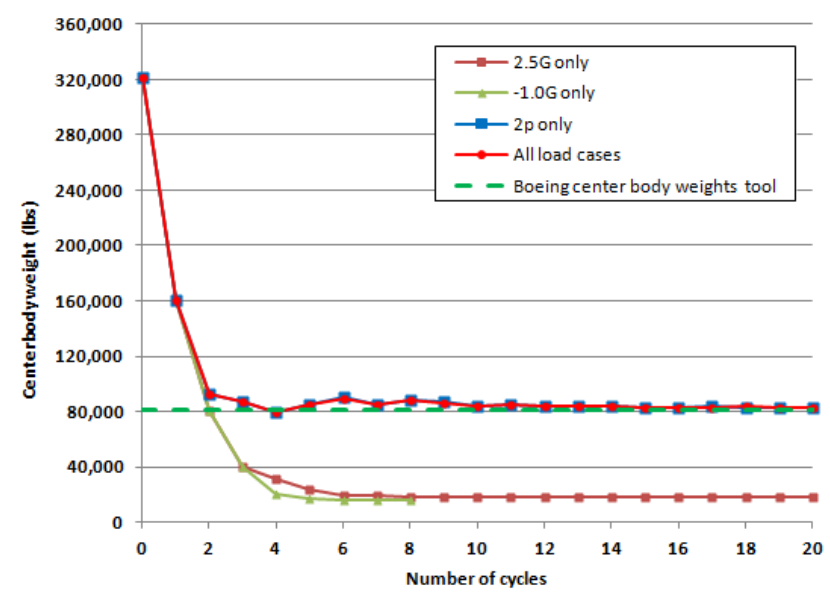

Figure 16: Convergence history for individual load cases vs. simultaneous optimization of all load cases.

Figure 17 shows deformation results for the three vs. five-bay design, and a comparison with Fig. 13 confirms that significant deflection relief is provided by the internal walls. For identical displacement constraints on the centerbody, the five bay design leads to a more lightweight configuration for the relatively large size of the validation cases. For smaller centerbody sizes, it will be shown in the scaling study results that little to no weight penalty is incurred when the design is fully stressed. Closer examination of the results then shows that material is redistributed between the internal wall panels.

Figure 18 shows that both multi-bay designs rapidly converge towards the structural weight of the validation case. The three-bay design results in a slightly higher weight as compared to the five-bay design due to the increased displacement relief provided by the additional internal walls.

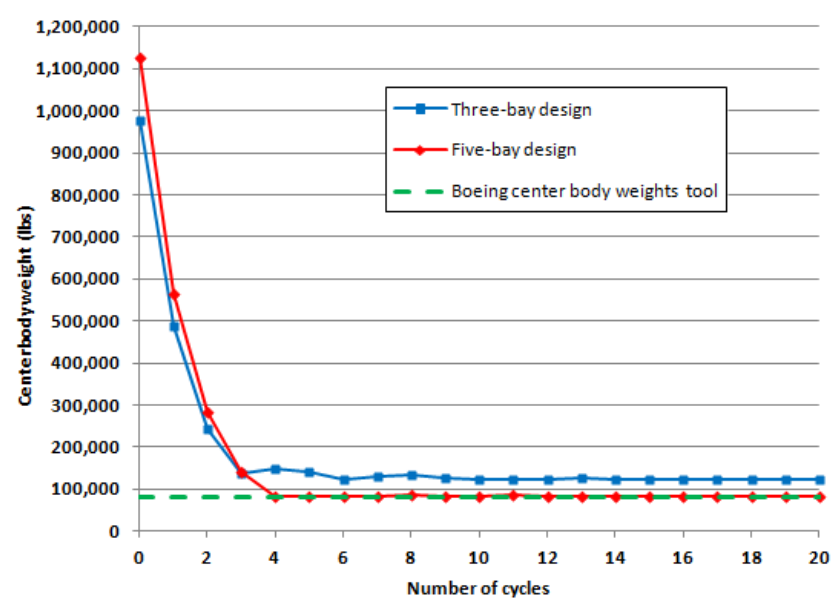

Figure 18: Convergence history for individual load cases vs. simultaneous optimization of all load cases. 
Figure 19 shows excellent agreement between both methods for several pitch angles from 1 to 4 degrees. Small differences between the sectional lift coefficients in the midsection of the HWB are attributable to differences in the geometry extraction and dihedral modeling between the Nastran and Vorview implementation.

Note that the Vorview spanload data is based on rigid lifting surfaces since Vorview does not employ an underlying structural model. Therefore, only the rigid sectional lift coefficients from the Nastran solution are shown for comparison.

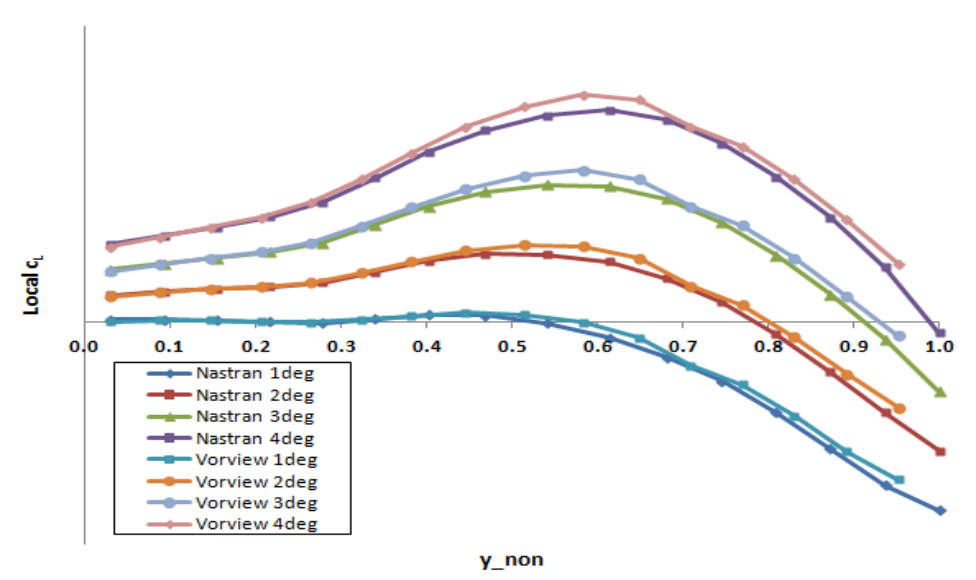

Figure 19: Spanload distributions vs. pitch angle from Nastran doublet lattice and Vorview vortex lattice aerodynamics.

\section{HWB Centerbody Scaling Study}

\section{A. HWB Scaling Study Background}

The FEM based centerbody weights tool was used to calculate centerbody structural weights in a scaling study by Nickol $^{13}$ for a family of HWB designs ranging from regional jet size (98pax) to very large twin-aisle jet size (400pax) as depicted in Fig. 20. Initial studies on HWB concepts focused on very large applications with capacities for up to 800 passengers. More recent studies have focused on the large, twin-aisle class with passenger capacities in the 300-450 range. Efficiently scaling the HWB concept down to single aisle or smaller sizes is challenging due to geometric constraints, potentially reducing the desirability of this concept for applications in the 100-200 passenger

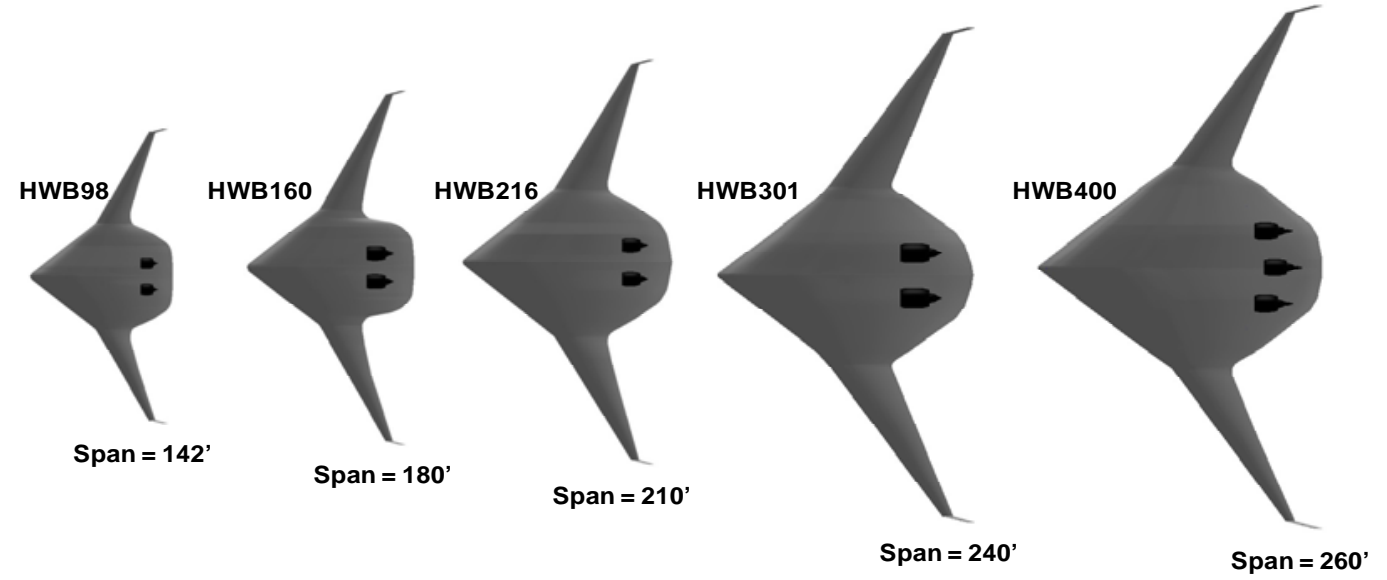

Figure 20: Scaled planform views of the HWB designs used in the scaling study. ${ }^{13}$

Table 1: Vehicle classes and nomenclature for HWB scaling study designs. ${ }^{13}$

\begin{tabular}{lcccccc}
\hline \hline Vehicles size class & $\begin{array}{c}\text { Number of } \\
\text { passengers }\end{array}$ & $\begin{array}{c}\text { Design range } \\
\text { (nmi) }\end{array}$ & $\begin{array}{c}\text { Cruise Mach } \\
\text { number }\end{array}$ & $\begin{array}{c}\text { Cruise } \\
\text { altitude (ft) }\end{array}$ & $\begin{array}{c}\text { Cruise lift } \\
\text { coefficient }\end{array}$ & Designation \\
\hline Regional jet & 98 & 2,400 & 0.78 & 39,000 & 0.17 & HWB98 \\
Large single aisle & 160 & 2,875 & 0.78 & 39,000 & 0.19 & HWB160 \\
Small twin aisle & 216 & 6,600 & 0.80 & 38,000 & 0.18 & HWB216 \\
Large twin aisle & 301 & 7,500 & 0.84 & 35,000 & 0.22 & HWB301 \\
Very large & 400 & 5,800 & 0.85 & 35,000 & 0.22 & HWB400 \\
\hline \hline
\end{tabular}


capacity range or less. In order to quantify this scaling challenge, five advanced conventional (tube-and-wing layout) concepts were compared to equivalent HWB concepts in terms of fuel burn performance.

The comparison showed that the HWB concepts have fuel burn advantages over advanced tube-and-wing concepts in the larger payload/range classes (roughly 767-sized and larger) while benefits are significantly reduced for the smaller HWB designs. More details regarding this scaling study can be found in Ref. 13. Planform renderings, design ranges, and cruise Mach numbers for the HWB designs used in the study are shown in Fig. 20. The respective HWB designation indicates the specific passenger size of the

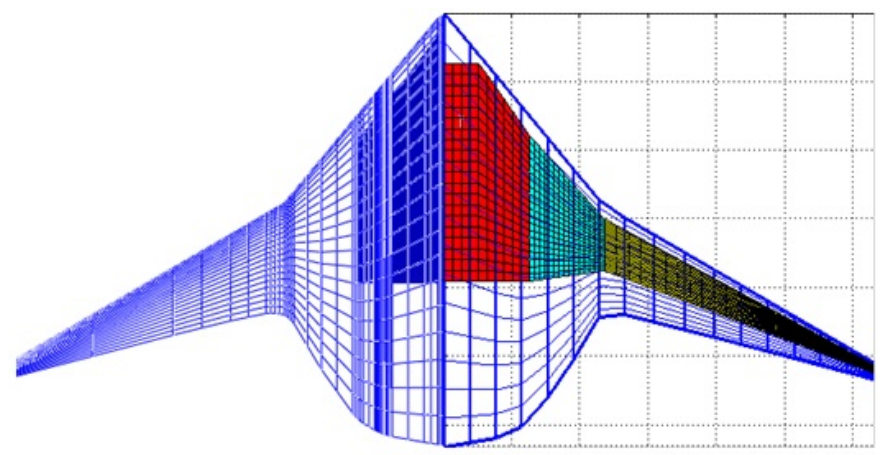

Figure 21: VSP model (left) and Nastran aeroelastic finite element model (right) for the HWB216 design. aircraft (e.g. HWB98 = 98pax, see Table 1). Cruise Mach number and altitude as indicated in Table 1 were used to derive the maneuver load conditions using the procedure described in section III.D.

The procedure described in section III was used to develop centerbody finite element models for all five HWB designs (Fig. 21). For the original centerbody design, VSP was used to fit seat arrangements and other features of the passenger cabin layout such as lavatories and galleys into the respective HWB design. Passenger height and landing gear requirements proved especially difficult for the smaller HWB vehicles.

Salient features of the centerbody structure were then used to define the home plate geometry data described in section III.A. Fig. 21 shows a comparison between the VSP based centerbody design (left half picture) and the resulting structural finite element model (right half picture) for the HWB216 design.

\section{B. HWB Scaling Study Centerbody Weights Results}

Centerbody structural weights were calculated for all five designs using the three- and five-bay design options. Figure 22 shows the centerbody weights from these analyses as compared to previous FLOPS results. While the finite element based results are generally slightly lower than the FLOPS based analysis, the three-bay design for the

Figure 22: Centerbody structural weight for three and five-bay designs. 


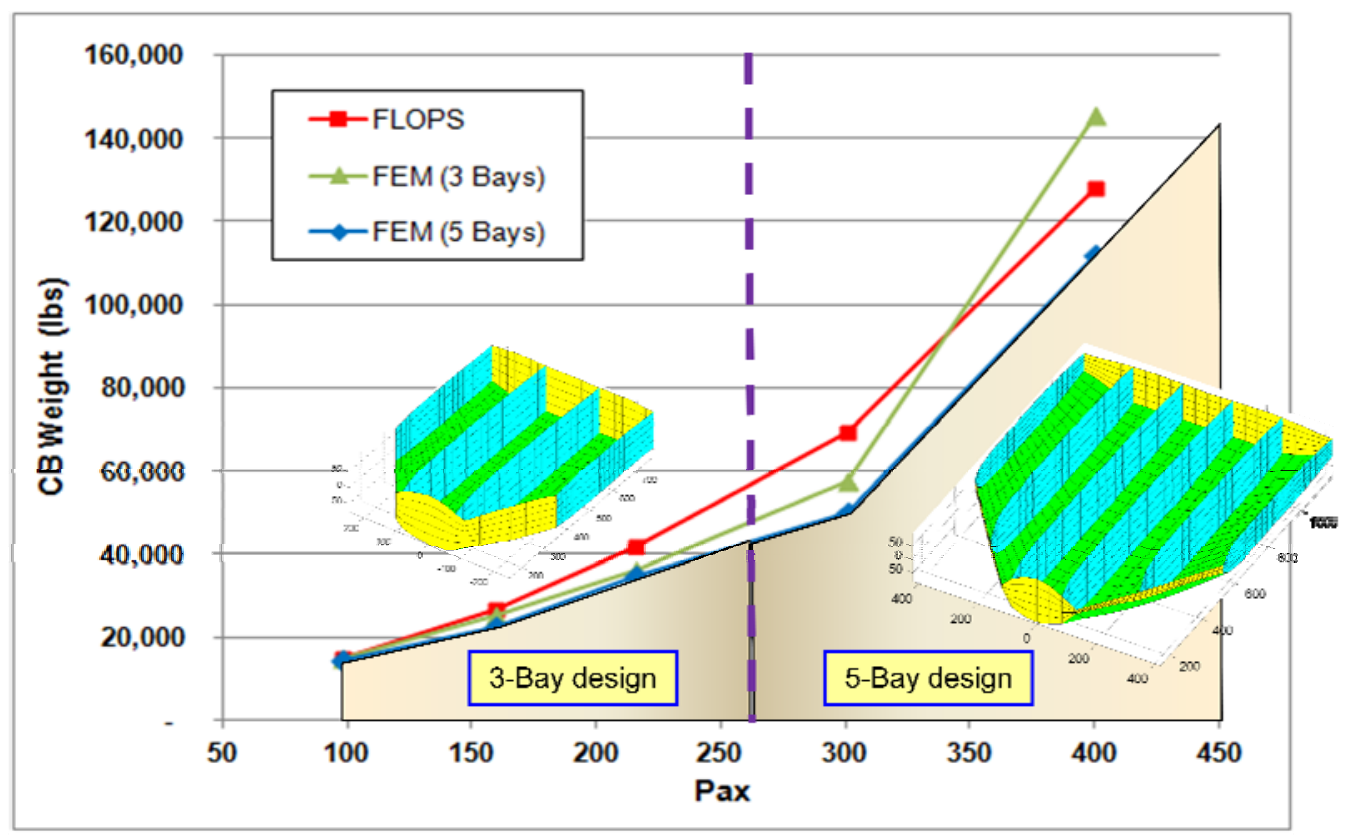

Figure 23: HWB Centerbody design space for three vs. five bay designs.

HWB400 shows a significant weight penalty as compared to both FLOPS and the five-bay design. This is due to the much stronger displacement relief provided by the five-bay design over the three-bay design. While this effect is also observable for the smaller HWB vehicles, it only becomes prominent for centerbody sizes upwards of 250pax.

The results indicate that for smaller HWB centerbodies, the internal wall arrangement is driven by passenger cabin layout requirements rather than by structural displacement constraints. This allows the designer more freedom in the internal layout for the smaller HWB vehicles, where cabin space is at a premium. Since displacement constraints are becoming prominent for larger centerbodies, switching to a five-bay design at this point will provide for significant structural weight reductions. Since the exact transition point from a three to a five-bay design depends on the actual centerbody design details, it is still recommended to perform the structural and weights analysis for both options of a specific design.

The results of the scaling study show that the present tool can not only be used to analyze specific centerbody configurations, but it can also provide important design guidance for preliminary design studies regarding the number of required internal walls for displacement relief and structural weight reduction.

Based on this study, the advantages of a five-bay design become significant for passenger cabins upwards of about 270pax; while a three-bay centerbody seems to be more practical for smaller HWB vehicles (Fig. 23).

\section{Conclusions}

This paper presents a new, fast, and scalable finite element model for structural analysis, optimization, and weight calculation of HWB centerbodies. The method is highly scalable and can be used to analyze any HWB centerbody based on a VSP model of the air vehicle and a set of predefined structural parameters. Since the model is based on a direct structural analysis rather than regression or approximation methods, weight results are also suitable for scaling studies or higher-risk "outlier" configurations. The high degree of scalability allows for flexible numbers of elements in the chordwise, spanwise, and thickness directions, resulting in variable model sizes, degrees of freedom, design variables, and design constraints, rendering this model the perfect choice for exploring design sensitivities and convergence studies.

The model has been validated using available data from preliminary design efforts by The Boeing Company, FLOPS centerbody weight results, aerodynamic data from X-48B wind tunnel testing, and Vorview vortex lattice analyses. The structural optimization is currently limited to calculating the centerbody weight, while leaving the sizing of the HWB midsection and outboard wing to FLOPS. This was done in an effort to gradually incorporate higher fidelity analyses into the current FLOPS optimization environment and to perform dedicated centerbody weight analyses for the HWB scaling study. However, the model is already set up to perform a structural analysis 
and optimization on the entire HWB primary structure, including centerbody, midsection, and outboard wing. For more in-depth analyses, the design model can easily be expanded to cover a larger variety of loads and analysis options, including gust loads, trim analyses, stability and control options, or flutter constraints.

Three different centerbody design options are available for a conceptual design level structural analysis and optimization. While the single-bay centerbody design does not account for accurate displacement constraints, it provides quick and accurate centerbody weight estimates. The multi-bay design options are representative of more detailed structural models developed during in-depth preliminary design studies. These models allow for the proper application of displacement constraints and are therefore recommended for analyses requiring higher confidence levels or for unconventional centerbodies where design sensitivities are unknown. The addition of internal walls provides significant displacement relief and has been shown to provide design guidance regarding the actual number of internal walls for more detailed preliminary design studies. The present scaling study indicates that the advantages of a five-bay design become significant for passenger cabin sizes upwards of about 270pax; while a three-bay centerbody is more practical for smaller HWB vehicles. However, the exact transition point from a three to a five-bay design depends on the specific centerbody design parameters. Therefore, it is recommended to perform the structural and weights analysis for both options and base the final layout decision on the obtained results.

\section{Acknowledgements}

The author would like to thank his co-workers Craig L. Nickol and Andrew S. Hahn at NASA Langley for their contributions and valuable discussions.

The presented work was supported by Dr. Fay Collier under the NASA Environmentally Responsible Aviation (ERA) project.

\section{References}

1 Liebeck, R.H., “Design of the BWB Subsonic Transport”, AIAA-2002-0002, 40 ${ }^{\text {th }}$ AIAA Aerospace Sciences Meeting and Exhibit, January 14-17, 2002, Reno, NV.

2 Risch, T., Cosentino, G., Regan, C.D., Kisska, M., and Princen, N., “X-48B Flight-Test Progress Overview,” AIAA-20090934, $47^{\text {th }}$ AIAA Aerospace Sciences Meeting and Exhibit, January 5-8, 2009, Orlando, Florida.

3 Gern, F.H., “Improved Aerodynamic Analysis for Hybrid Wing Body Conceptual Design Optimization,” AIAA-2012-0249, $50^{\text {th }}$ AIAA Aerospace Sciences Meeting and Exhibit, January 9-12, 2012, Nashville, Tennessee.

4 McCullers, L. A., "FLOPS Flight Optimization System, FLOPS manual.doc,” FLOPS User Manual, updated Dec. 2009.

5 Nickol, C.L. and McCullers, L.A., "Hybrid Wing Body Configuration System Studies,” AIAA-2009-0931, 47th AIAA Aerospace Sciences Meeting and Exhibit, January 5-8, 2009, Orlando, Florida.

$6 \quad$ McCullers, L. A.: "FLOPS Weight Module Documentation, Wate.doc,” FLOPS Users Manual, April 2008.

7 Bradley, K.R., “A Sizing Methodology for the Conceptual Design of Blended-Wing-Body Transports,” NASA/CR-2004213016, NASA Langley Research Center, Hampton, Virginia, September 2004.

8 Hahn, A., "Vehicle Sketch Pad: A Parametric Geometry Modeler for Conceptual Aircraft Design,” 48th AIAA Aerospace Sciences Meeting and Exhibit, January 4-7, 2010, Orlando, Florida.

9 Murchison, L., “A Method for The Parametric Analysis of Blended Wing Body Aircraft,” Student Career Experience Program Report, Aeronautics Systems Analysis Branch, Systems Analysis and Concepts Directorate, NASA Langley Research Center, Hampton, VA, December 2010.

$10 \mathrm{Li}, \mathrm{V}$. and Velicki, A., “Advanced PRSEUS Structural Concept Design and Optimization,” AIAA-2008-5840, 12th AIAA/ISSMO Multidisciplinary Analysis and Optimization Conference, 10-12 September 2008, Victoria, British Columbia, Canada.

11 Velicki, A., Thrash, P., and Jegley, D., “Airframe Development for the Hybrid Wing Body Aircraft,” AIAA-2009-932, 47th AIAA Aerospace Sciences Meeting Including The New Horizons Forum and Aerospace Exposition, January 5-8, 2009, Orlando, Florida.

12 Yovanof, N.P., Velicki, A., and Li, V., “Advanced Structural Stability Analysis of a Noncircular, BWB-Shaped Vehicle,” AIAA-2009-2452, 50th AIAA/ASME/ASCE/AHS/ASC Structures, Structural Dynamics, and Materials Conference, May 4-7, 2009, Palm Springs, California.

13 Nickol, C.L., “Hybrid Wing Body Configuration Scaling Study,” AIAA-2012-0337, 50 ${ }^{\text {th }}$ AIAA Aerospace Sciences Meeting and Exhibit, January 9-12, 2012, Nashville, Tennessee.

14 MSC Nastran 2008, Quick Reference Guide, MSC Software Corporation, 2 MacArthur Place, Santa Ana, CA 92707, USA, 2008, p. 2077.

15 Rodden, W.P. and Johnson, E.H. (Editors), MSC.Nastran Version 68, Aeroelastic Analysis User’s Guide, MSC Software. 
16 Vicroy, D.D., “Blended-Wing-Body Low-Speed Flight Dynamics: Summary of Ground Tests and Sample Results,” AIAA2009-0933, 47 ${ }^{\text {th }}$ AIAA Aerospace Sciences Meeting and Exhibit, January 5-8, 2009, Orlando, Florida.

17 Carter, M.B., Vicroy, D.D., and Patel, D., "Blended-Wing-Body Transonic Aerodynamics: Summary of Ground Tests and Sample Results,” AIAA-2009-0935, 47 ${ }^{\text {th }}$ AIAA Aerospace Sciences Meeting and Exhibit, January 5-8, 2009, Orlando, Florida.

18 Velicki, A., Damage Arresting Composites for Shaped Vehicles, NASA/CR-2009-215932, The Boeing Company, Huntington Beach, California, NASA Langley Research Center, Hampton, Virginia, 23681-2199, September 2009.

19 MSC Nastran 2005 r3, Design Sensitivity and Optimization, User’s Guide, MSC Software Corporation, 2 MacArthur Place, Santa Ana, CA 92707, USA, 2005.

20 Miranda, L.R., Elliott, R.D., and Baker, W.M., A Generalized Vortex Lattice Method for Subsonic and Supersonic Flow Applications, NASA-CR-2865, Lockheed-California Company, Burbank, CA, December 1977.

21 Vorview Documentation, Vorview 1.7.4, 1999, contact: A. Hahn, Aeronautics Systems Analysis Branch, NASA Langley Research Center, Mail Stop 442, Hampton, VA 23681-0001. 